\title{
EXTENSION THEOREMS FOR NONLINEAR DISJOINTLY ADDITIVE FUNCTIONALS AND OPERATORS ON LEBESGUE SPACES, WITH APPLICATIONS
}

\author{
BY MOSHE MARCUS ${ }^{1}$ AND VICTOR J. MIZEL ${ }^{1}$ \\ Communicated by Robert Bartle, October 17, 1975
}

In recent years there has been developed a reasonably complete representation theory for nonlinear disjointly additive functionals defined on various Banach spaces of measurable functions [1], [6], [7]. Such functionals, which are involved in many nonlinear integral and differential equations, share some important regularity properties of linear functionals [4, Chapter 5]. Hence the question naturally arises as to whether a Hahn-Banach type extension theory is available for disjointly additive functionals . It turns out that a full analogue of the Hahn-Banach theorem does not hold, but there are important classes of subspaces for which an extension result is available. As a byproduct of this extension result one obtains a representation formula for all continuous disjointly additive functionals defined on such subspaces. It should be noted that a representation result of this type cannot be obtained by the methods used in the above cited works, since these subspaces are not complete lattices while the completeness of the lattice structure was essential in the arguments used previously. Among the applications stemming from this extension theory is a characterization of those nonlinear functionals on Sobolev spaces $W_{k}^{p}(J)(1 \leqslant p \leqslant \infty, k \geqslant 1)$ which have the form:

$$
N(u)=\int_{J} H\left(t, D^{k}(u(t))\right) d t, \quad u \in W_{k}^{p}(J),
$$

where $J$ is a subinterval of the line and $H: J \times R \rightarrow R$ is a function obeying the Carathéodory conditions [5].

Let $(\Omega, T, m)$ be a finite nonatomic measure space. We say that a closed subspace $M \subset L^{p}(m)(1 \leqslant p \leqslant \infty)$ possesses the $d$. a. extension property if every continuous functional $N: M \rightarrow R$ which is disjointly additive in the sense.

$$
N(u, v)=N(u)+N(v), \quad \forall u, v \in M \text { s.t. } u v=0,
$$

has an extension to $L^{p}(m)$ which preserves these properties. For the case $p=\infty$, the continuity here is to be taken relative to the topology generated by bounded sequential convergence in measure, in brief the $(\mathrm{bm})$ topology. Call a closed linear subspace $M \subset L^{\infty}(m)$ a rich subspace if: (a) $M$ is $w^{*}$-closed, (b) $M$ separates

AMS (MOS) subject classifications (1970). Primary 46E35, 46G99; Secondary 28A15.

${ }^{1}$ Partially supported by NSF Grant MPS 71-02776-A03.

Copyright (c) 1976, American Mathematical Society 
sets, in the sense that for every nonnull set $E \in T$ there exists a nonnull function $f \in M$ such that $f=f X_{E}$. Call a closed linear subspace $M \subset L^{p}(m)(1 \leqslant p<\infty)$ a rich subspace if $M \cap L^{\infty}(m)$ is a rich subspace of $L^{\infty}(m)$. (This notion is closely related to the notion of a thin set in $L^{1}(m)$ given in [2] .) For $1 \leqslant p$ $<\infty$, every closed subspace of finite codimension is rich; for $p=\infty$, every $w^{*}$. closed subspace of finite codimension is rich. There also exist rich subspaces of infinite codimension.

THEOREM A. (1) If $M \subset L^{\infty}(m)$ is a rich subspace then it possesses the d.a. extension property.

(2) If $M \subset L^{p}(m)(1 \leqslant p<\infty)$ is of finite codimension then it possesses the d.a. extension property.

REMARKS. We conjecture that (2) remains valid even if its hypothesis is weakened to " $M$ is a rich subspace".

A function $H: \Omega \times R \rightarrow R$ which satisfies the Carathéodory conditions gives rise to a transformation $\mathbf{H}$ via

$$
(\mathrm{H} v)(x)=H(x, v(x)), \quad x \in \Omega
$$

Write $H \in \operatorname{Car}^{p}(\Omega)(1 \leqslant p \leqslant \infty)$ provided that $\mathbf{H} v$ is in $L^{1}(m)$ for all $v \in L^{p}(m)$. The function $H$ is normalized if $H(\cdot, 0)=0$ a.e.

Theorem B. Suppose that $M \subset L^{p}(m)(1 \leqslant p \leqslant \infty)$ satisfies the hypothesis in Theorem A and let $N: M \rightarrow R$ be disjointly additive and continuous $[(\mathrm{bm})$-continuous, if $p=\infty]$. Then there exists a normalized function $H \in$ $\operatorname{Car}^{p}(\Omega)$ such that

$$
N(u)=\int_{\Omega} \mathbf{H} u d m, \quad u \in M
$$

Moreover a second normalized function $H^{*} \in \operatorname{Car}^{p}(\Omega)$ satisfies (*) if and only if

$$
H^{*}(x, a)-H(x, a)=a g(x), \quad a \in R, x \in \Omega,
$$

where $g$ is an arbitrary element in the $L^{q}$-annihilator of $M$, where $1 / p+1 / q=1$.

Similar extension results are available for continuous transformations $H: M$ $\rightarrow L^{1}(m)$ which are disjointly additive and local in the sense that $H u$ vanishes wherever $u$ vanishes.

We proceed to give some indication of the concepts and methods involved. With every rich subspace $M \subset L^{p}(m)$ one can associate a vector valued measure $\mu$ of Lyapunov type [3] such that $M$ consists precisely of those functions of $\mu$ mean zero. An important step is the following Radon-Nikodym type result which is of interest in its own right. 
Lemma. Let $M \subset L^{\infty}(m)$ be a rich subspace and let $\Lambda$ be a (bm)continuous odd, disjointly additive functional whose domain is given by: $E_{M}=$ $\{f \in M:$ range $f \subset\{0,1,-1\}\}$. Then there exists $G \in L^{1}(m)$ such that

$$
\Lambda(f)=\int_{\Omega} G f d m, \quad f \in E_{M} .
$$

Proofs and related results will appear elsewhere.

\section{REFERENCES}

1. L. Drewnowski and W. Orlicz, Continuity and representation of orthogonally additive functionals, Bull. Acad. Polon. Sci., Sér. Sci. Math. Astronom. Phys. 17 (1969), 647-653. MR 41 \#806.

2. J. F. C. Kingman and A. P. Robertson, On a theorem of Lyapunov, J. London Math. Soc. 43 (1968), 347-351. MR 37 \%67.

3. I. Kluvanek, Lectures on vector measures, Lecture notes, Van Nostrand Mathematical Studies (to appear).

4. M. A. Krasnosel'skiř, P. P. Zabreĭko, et al., Integral operators in spaces of summable functions, "Nauka", Moscow, 1966; English transl., Noordhoff, Groningen, 1975. MR 34 \#6568.

5. M. Marcus and V. J. Mizel, Representation theorems for non-linear disjointly additive functionals on Sobolev spaces (to appear).

6. V. J. Mizel, Characterization of non-linear transformations possessing kernels, Canad. J. Math. 22 (1970), 449-471. MR 41 \#7495.

7. V. J. Mizel and K. Sundaresan, Representation of vector-valued non-linear functions, Trans. Amer. Math. Soc. 159 (1971), 111-127. MR 43 \#5368.

DEPARTMENT OF MATHEMATICS, CARNEGIE-MELLON UNIVERSITY, PITTSBURGH, PENNSYLVANIA 15213

Current address (Victor J. Mizel): Department of Mathematics, Technion, Haifa, Israel 